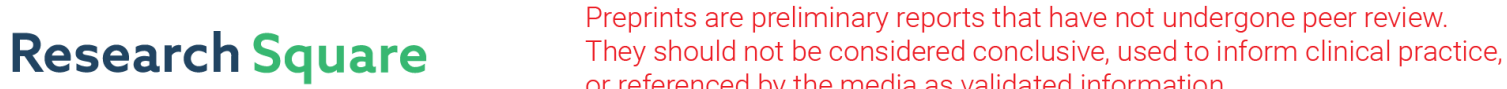 or referenced by the media as validated information. \\ Nonlinear Control Design for Walking Assistance Training Robotic Systems
}

Wei Liu ( $\square$ intervalm@163.com )

Kochi University of Technology https://orcid.org/0000-0001-7116-510X

\section{Shuoyu Wang}

Kochi University: Kochi Daigaku

\section{Peng Shi}

The University of Adelaide

\section{Research Article}

Keywords: State feedback control, walking assistance training robot, nonlinear dynamical system, control input, tilt angle.

Posted Date: February 1st, 2022

DOI: https://doi.org/10.21203/rs.3.rs-1203620/v1

License: @ (i) This work is licensed under a Creative Commons Attribution 4.0 International License. Read Full License 


\title{
Nonlinear Control Design for Walking Assistance Training Robotic Systems
}

\author{
Wei Liu, Shuoyu Wang, and Peng Shi
}

\begin{abstract}
In this paper, the control problem for a novel walking assistance training robot is considered where the main objective is utilizing the robot to control the tilt angle and the position of a user. First, the novel walking assistance training robot together with a user is modeled as a nonlinear dynamical system in state space form with control input. Then, based on the nonlinear dynamical system and some results proposed in this paper, a feedback control system is developed, and a nonlinear state feedback control algorithm is designed where the control input includes two parts, namely, a dynamical part and a constant part. By doing this, it is ensured that the feedback control system is stable, and achieves the main objective under some limits for the control input. A simulation example is given to illustrate the effectiveness and performance of the proposed nonlinear state feedback control algorithm.
\end{abstract}

\section{Index Terms}

State feedback control, walking assistance training robot, nonlinear dynamical system, control input, tilt angle.

\section{INTRODUCTION}

Over the past few decades, a lot of efforts have been made to utilize robotic technologies to improve walking function, fitness, and quality-of-life among people having illness, disability, injury or other health condition that influences the ability to walk [1]-[6]. A robot with such a

This work was partially supported by the National Nature Science Foundation of China (62073125, 61773131), the DongGuan Innovative Research Team Program (2020607202006), and the Australian Research Council (DP170102644).

Wei Liu is with the Research Institute, Kochi University of Technology, Kochi 782-8502, Japan, and also with the School of Information and Electronic Engineering, Zhejiang Gongshang University, Hangzhou 310018, China (e-mail: intervalm@163.com).

Shuoyu Wang is with the School of Systems Engineering, Kochi University of Technology, Kochi 782-8502, Japan (e-mail: wang.shuoyu@kochi-tech.ac.jp).

Peng Shi is with the School of Electrical and Electronic Engineering, the University of Adelaide, SA 5005, Australia (e-mail: peng.shi@adelaide.edu.au). 
function is called walking assistance training robot (WATR). Until now, a rich and diverse body of literature has emerged on the design, analysis and control of WATRs in a wide variety of structures. A major class of WATRs focuses on designing power-assisted cart/walker to achieve the purpose of walking assistance [7]-[11]. In [7], an adaptive shared control system was presented to monitor the mobility and health of the elderly who was using a walker configuration. Based on passive robotics, an intelligent walker was developed in [8] where the walker includes two casters, a support frame and two wheels equipped with servo brakes. An interactive control method for robotic walker was presented in [9], which can control the direction and velocity of the walker according to the user's movement behaviors. In [10], a robot was developed for walking training, and a three-stage approach was described to conduct function and user tests. In [11], a path following strategy with a mechanical braking guidance was proposed, which can guide the user follow the planned path.

Another important part for the design of WATRs is based on crunch [12]-[14] and cane [15], [16]. An instrumented Lofstrand crutch system was developed in [12] utilizing an inverse dynamics model, which uses four, six-degree-of-freedom force transducers to obtain threedimensional upper extremity joint reaction forces. In [13], the authors designed two wireless instrumented crutches for gait monitoring, and the characterization of the crutches was described. A measurement system called as the InSight crutches was presented in [14], which allows to connect with wearable robots. In [15], using a stick, an omnidirectional basis and a group of sensors, the authors designed an intelligent cane robot for recognizing the user's walking intention, and proposed a control scheme for admittance motion based on the cane robot. In terms of an inverted pendulum model and the Lie algebra method, a robotic cane was proposed in [16] to help the body maintain balance. In recent years, wearable robot has become popular and has attracted increasing attention from researchers [17]-[21]. In [17], using a double-layered wire for transferring the power, a tendon-driven wearable exoskeleton was proposed, and the torque mode control was discussed based on the fuzzy method. In [18], the authors proposed an alternative motion planning approach so that the wearable robot can generate various stride length and walking speed. In [19], a powered exoskeleton with functional electrical stimulation was described as a hybrid system, and a control scheme was presented using two control loops. Applying a data-driven learning approach, assistive strategies for interactions of users and exoskeleton robots are designed in [20]. In [21], the authors proposed an individualized gait pattern generation model, and the model was applied to exoskeleton robot. More results for 
WATRs and related control methods can be found in [22]-[29] and references therein.

In this paper, different from the structure of the existing WATRs, a novel design for WATR is developed. In this design, the robot's user follows the robot with the help of two straps, and we want to control the user's tilt angle and position via the two straps under some limits for the forces on the two straps. At the same time, the two straps can reduce the load on the user's legs and hip joint according to the user's clinical and physical circumstances. In order to achieve the aim, we first present a nonlinear dynamical model in state space form with control input to describe the novel WATR together with a user. Then, we develop a feedback control system based on the presented nonlinear dynamical model, and we propose a nonlinear state feedback control algorithm to control the pulling forces on the two straps so that the user's tilt angle and position can be controlled to the desired values. Hence, the novel design of WATR is useful for correcting the posture of users with various demands, and is promising to further improve the quality of posture correction in contrast to the existing WATRs.

The remainder of this paper is organized as follows. Section II formulates system modeling and the control problem under consideration. In Section III, a feedback control system is developed and a nonlinear state feedback control algorithm is designed. In Section IV, a simulation example is given to illustrate the performance of the developed control algorithm. The conclusion is given in Section V.

\section{System Modeling And Problem Formulation}

Consider the WATR whose structure is given in Fig. 1 where $L_{1}$ and $L_{2}$ are two straps attached to the chest and back, respectively, of the user; $f_{1}$ and $f_{2}$ are pulling forces on $L_{1}$ and $L_{2}$, respectively; $\alpha$ is the angle between $y$ and $L_{1} ; \beta$ is the angle between $y$ and $L_{2} ; \theta$ is the user's tilt angle; and $l_{1}$ and $l_{2}$ stand for the distances between the center of gravity of the user and outside of the body in different directions. Using Newton's second law, the equations of motion in $x$ and $y$ directions can be written as

$$
\begin{array}{r}
f_{1}(t) \sin \alpha(t)-f_{2}(t) \sin \beta(t)=m \ddot{x}(t), \\
-f_{1}(t) \cos \alpha(t)-f_{2}(t) \cos \beta(t)-f_{p}+m g=m \ddot{y}(t)
\end{array}
$$

where $m$ is the mass of the user; $\ddot{x}=a_{x}$ is the acceleration in $x$ direction; $f_{p}$ is the force on the supporting legs of the user; $g$ is the gravitational acceleration; and $\ddot{y}=a_{y}$ is the acceleration 


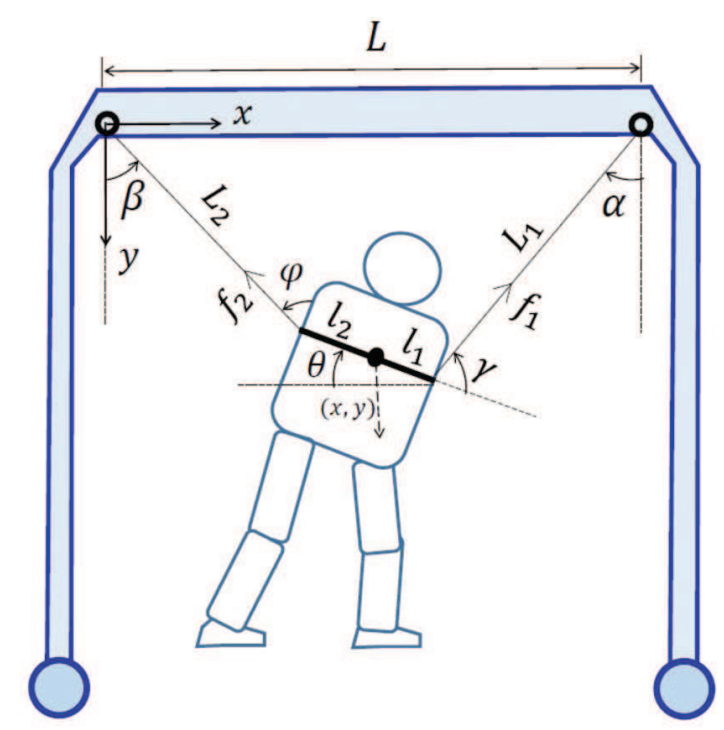

Fig. 1. Structure of the novel WATR.

in $y$ direction. Because it is hard for the user to bear the weight of the whole body during the rehabilitation period, we take

$$
f_{p}=\varepsilon m g
$$

with $0 \leq \varepsilon \leq 1$ where $\varepsilon$ is obtained from the user's clinical and physical circumstances. Substituting (3) into (2), we get

$$
-f_{1}(t) \cos \alpha(t)-f_{2}(t) \cos \beta(t)+(1-\varepsilon) m g=m \ddot{y}(t) .
$$

The moment equation can be written as

$$
-f_{1}(t) l_{1}(t) \sin \gamma(t)+f_{2}(t) l_{2}(t) \cos \varphi(t)=I \ddot{\theta}(t)
$$

where $\varphi$ and $\gamma$ are given in Fig. 1 ; $I$ is the moment of inertia; and $\ddot{\theta}=a_{\theta}$ is the angular acceleration. From Fig. 1, we see that

$$
\begin{aligned}
\varphi(t) & =\beta(t)+\theta(t), \\
\gamma(t) & =\frac{\pi}{2}-\psi(t) .
\end{aligned}
$$

with

$$
\psi(t) \triangleq \alpha(t)-\theta(t)
$$


Substitute (7) into (5) to obtain

$$
-f_{1}(t) l_{1}(t) \sin \left(\frac{\pi}{2}-\psi(t)\right)+f_{2}(t) l_{2}(t) \cos \varphi(t)=I \ddot{\theta}(t)
$$

that is,

$$
-f_{1}(t) l_{1}(t) \cos \psi(t)+f_{2}(t) l_{2}(t) \cos \varphi(t)=I \ddot{\theta}(t)
$$

Because the center of gravity of the user is in the middle, we have

$$
l_{1}(t)=l_{2}(t)=0.5 l
$$

where $l$ is the shoulder width of the user. Substituting (10) into (9) yields that

$$
-0.5 f_{1}(t) l \cos \psi(t)+0.5 f_{2}(t) l \cos \varphi(t)=I \ddot{\theta}(t) .
$$

Let $v_{x}$ and $v_{y}$ be the velocities in $x$ and $y$ directions, respectively, and let $v_{\theta}$ be the angular velocity. Then, we easily obtain

$$
\dot{x}=v_{x}, \dot{y}=v_{y}, \dot{\theta}=v_{\theta}
$$

Using (1), (4), (11) and (12), we obtain the following equation

$$
A_{m} \dot{\chi}(t)=A_{1} \chi(t)+B_{1}(t) f(t)+d_{1}
$$

where

$$
\begin{aligned}
& A_{m}=\left(\begin{array}{cccccc}
1 & 0 & 0 & 0 & 0 & 0 \\
0 & m & 0 & 0 & 0 & 0 \\
0 & 0 & 1 & 0 & 0 & 0 \\
0 & 0 & 0 & m & 0 & 0 \\
0 & 0 & 0 & 0 & 1 & 0 \\
0 & 0 & 0 & 0 & 0 & I
\end{array}\right), \chi(t)=\left(\begin{array}{c}
x(t) \\
v_{x}(t) \\
y(t) \\
v_{y}(t) \\
\theta(t) \\
v_{\theta}(t)
\end{array}\right), A_{1}=\left(\begin{array}{cccccc}
0 & 1 & 0 & 0 & 0 & 0 \\
0 & 0 & 0 & 0 & 0 & 0 \\
0 & 0 & 0 & 1 & 0 & 0 \\
0 & 0 & 0 & 0 & 0 & 0 \\
0 & 0 & 0 & 0 & 0 & 1 \\
0 & 0 & 0 & 0 & 0 & 0
\end{array}\right), \\
& B_{1}(t)=\left(\begin{array}{cc}
0 & 0 \\
\sin \alpha(t) & -\sin \beta(t) \\
0 & 0 \\
-\cos \alpha(t) & -\cos \beta(t) \\
0 & 0 \\
-0.5 l \cos \psi(t) & 0.5 l \cos \varphi(t)
\end{array}\right), d_{1}=\left(\begin{array}{c}
0 \\
0 \\
0 \\
(1-\varepsilon) m g \\
0 \\
0
\end{array}\right), f(t)=\left(\begin{array}{c}
f_{1}(t) \\
f_{2}(t)
\end{array}\right) .
\end{aligned}
$$


Multiplying both sides of (13) by $A_{m}^{-1}$, we have

$$
\dot{\chi}(t)=A \chi(t)+B(t) f(t)+d m g
$$

where

$$
\begin{aligned}
& A=A_{m}^{-1} A_{1}=\left(\begin{array}{cccccc}
0 & 1 & 0 & 0 & 0 & 0 \\
0 & 0 & 0 & 0 & 0 & 0 \\
0 & 0 & 0 & 1 & 0 & 0 \\
0 & 0 & 0 & 0 & 0 & 0 \\
0 & 0 & 0 & 0 & 0 & 1 \\
0 & 0 & 0 & 0 & 0 & 0
\end{array}\right) \\
& B(t)=A_{m}^{-1} B_{1}(t)=\left(\begin{array}{cc}
0 & 0 \\
\sin \alpha(t) / m & -\sin \beta(t) / m \\
0 & 0 \\
-\cos \alpha(t) / m & -\cos \beta(t) / m \\
0 & 0 \\
-0.5 l \cos \psi(t) / I & 0.5 l \cos \varphi(t) / I
\end{array}\right), d=A_{m}^{-1} d_{1}=\left(\begin{array}{c}
0 \\
0 \\
0 \\
(1-\varepsilon) g \\
0 \\
0
\end{array}\right) .
\end{aligned}
$$

For dynamical system described by (15), the paper aims to design a control algorithm via control input $f(t)$ such that the following requirements are simultaneously satisfied:

1) For the user's tilt angle $\theta(t)$, it satisfies $\lim _{t \rightarrow \infty} \theta(t)=\bar{\theta}$ where $\bar{\theta}$ is selected according to the actual status of the user.

2) The pulling forces $f_{1}(t)$ and $f_{2}(t)$ in the control input $f(t)$ satisfy $0 \leq f_{1}(t) \leq f_{1}^{\nabla}$ and $0 \leq f_{2}(t) \leq f_{2}^{\nabla}$ where $f_{1}^{\nabla}$ and $f_{2}^{\nabla}$ are determined in terms of the user's clinical and physical circumstances.

3) Let $\bar{x}$ and $\bar{y}$ be the values of $x(t)$ and $y(t)$, respectively, which is the most beneficial to the user in rehabilitation training. It holds that $\lim _{t \rightarrow \infty} x(t)=\bar{x}$ and $\lim _{t \rightarrow \infty} y(t)=\bar{y}$.

4) The load on the user's legs and hip joint can be reduced according to the user's clinical and physical circumstances.

\section{Main Results}

In order to control the user's tilt angle and satisfy the requirements presented in the previous section, we first propose the following theorem. 
Theorem 1: Under the condition that $\theta(t)=\bar{\theta}, \alpha(t)=\bar{\alpha}$ and $\beta(t)=\bar{\beta}$, as well as $\ddot{y}(t)=0$ and $\ddot{\theta}(t)=0$ where $\bar{\theta}$ is the desired values given in the previous section, and $\bar{\alpha}$ and $\bar{\beta}$ are given according to the actual requirements, it holds that $f_{1}(t)=\bar{f}_{1}$ and $f_{2}(t)=\bar{f}_{2}$ where $\bar{f}_{1}$ and $\bar{f}_{2}$ are computed according to

$$
\begin{aligned}
\bar{f}_{1} & =\frac{(1-\varepsilon) m g \cos (\bar{\beta}+\bar{\theta})}{\cos (\bar{\beta}+\bar{\theta}) \cos \bar{\alpha}+\cos (\bar{\alpha}-\bar{\theta}) \cos \bar{\beta}} \\
\bar{f}_{2} & =\frac{(1-\varepsilon) m g \cos (\bar{\alpha}-\bar{\theta})}{\cos (\bar{\beta}+\bar{\theta}) \cos \bar{\alpha}+\cos (\bar{\alpha}-\bar{\theta}) \cos \bar{\beta}} .
\end{aligned}
$$

Proof: Substitute $\alpha(t)=\bar{\alpha}, \beta(t)=\bar{\beta}$ and $\ddot{\theta}(t)=0$ into (4) to get

$$
-f_{1}(t) \cos \bar{\alpha}-f_{2}(t) \cos \bar{\beta}+(1-\varepsilon) m g=0
$$

which means that

$$
f_{1}(t)=\frac{(1-\varepsilon) m g-f_{2}(t) \cos \bar{\beta}}{\cos \bar{\alpha}} .
$$

Substituting $\theta(t)=\bar{\theta}$ and $\beta(t)=\bar{\beta}$ into (6) yields that

$$
\varphi(t)=\bar{\beta}+\bar{\theta}
$$

Substituting $\alpha(t)=\bar{\alpha}$ and $\theta(t)=\bar{\theta}$ into (8), we have

$$
\psi(t)=\bar{\alpha}-\bar{\theta}
$$

Substituting (20), (21) and $\ddot{\theta}(t)=0$ into (11), we get

$$
-0.5 f_{1}(t) l \cos (\bar{\alpha}-\bar{\theta})+0.5 f_{2}(t) l \cos (\bar{\beta}+\bar{\theta})=0,
$$

or equivalently,

$$
-f_{1}(t) \cos (\bar{\alpha}-\bar{\theta})+f_{2}(t) \cos (\bar{\beta}+\bar{\theta})=0 .
$$

Substitute (19) into (22) to derive

$$
-\frac{\left((1-\varepsilon) m g-f_{2}(t) \cos \bar{\beta}\right) \cos (\bar{\alpha}-\bar{\theta})}{\cos \bar{\alpha}}+f_{2}(t) \cos (\bar{\beta}+\bar{\theta})=0
$$

which means 


$$
f_{2}(t)=\frac{(1-\varepsilon) m g \cos (\bar{\alpha}-\bar{\theta})}{\cos (\bar{\beta}+\bar{\theta}) \cos \bar{\alpha}+\cos (\bar{\alpha}-\bar{\theta}) \cos \bar{\beta}} .
$$

Substitute (23) into (19) and rearrange to obtain

$$
f_{1}(t)=\frac{(1-\varepsilon) m g \cos (\bar{\beta}+\bar{\theta})}{\cos (\bar{\beta}+\bar{\theta}) \cos \bar{\alpha}+\cos (\bar{\alpha}-\bar{\theta}) \cos \bar{\beta}} .
$$

Putting (24) and (23) together, we prove the statement.

Remark 1: In order to obtain $\alpha(t)=\bar{\alpha}$ and $\beta(t)=\bar{\beta}$, we see from (1) that we need to take

$$
\frac{f_{1}(t)}{f_{2}(t)}=\frac{\sin \bar{\beta}}{\sin \bar{\alpha}}
$$

such that $\ddot{x}(t)=0$, that is, the user can keep the current state without using any extra force.

Remark 2: From Theorem 1, we see that, in order to obtain the desired control parameters presented in Theorem $1, \bar{f}_{1}$ and $\bar{f}_{2}$ should satisfy

$$
\frac{\bar{f}_{1}}{\bar{f}_{2}}=\frac{\cos (\bar{\beta}+\bar{\theta})}{\cos (\bar{\alpha}-\bar{\theta})} .
$$

From (25) and (26), it follows that

$$
\frac{f_{1}(t)}{f_{2}(t)} \neq \frac{\bar{f}_{1}}{\bar{f}_{2}}
$$

unless $\frac{\sin \bar{\beta}}{\sin \bar{\alpha}}=\frac{\cos (\bar{\beta}+\bar{\theta})}{\cos (\bar{\alpha}-\bar{\theta})}$, which means $\ddot{x}(t) \neq 0$ in the vast majority of cases when we take $f_{1}(t)=\bar{f}_{1}$ and $f_{2}(t)=\bar{f}_{2}$ so that the desired control parameters presented in Theorem 1 are satisfied. Hence, the user must utilize extra force to keep the current state when $\bar{f}_{1}$ and $\bar{f}_{2}$ are utilized to obtain the desired control parameters presented in Theorem 1. As a reasonable compromise, we only take one of the values among $\bar{\alpha}$ and $\bar{\beta}$. Without loss of generality, we take one value $\beta(t)=\bar{\beta}$.

After a compromise for the selection of $\alpha(t)$, we propose the following theorem.

Theorem 2: Under the condition that $\theta(t)=\bar{\theta}$ and $\beta(t)=\bar{\beta}$, as well as $\ddot{x}(t)=0, \ddot{y}(t)=0$ and $\ddot{\theta}(t)=0$, it holds that $\alpha(t)=\vec{\alpha}, f_{1}(t)=\vec{f}_{1}$ and $f_{2}(t)=\vec{f}_{2}$ with

$$
\begin{aligned}
\vec{\alpha} & =\arcsin \left(\frac{\sin \bar{\beta} \cos \bar{\theta}}{\sqrt{(\cos \vec{\varphi}-\sin \bar{\beta} \sin \bar{\theta})^{2}+\sin ^{2} \bar{\beta} \cos ^{2} \bar{\theta}}}\right), \\
\vec{f}_{1} & =\frac{(1-\varepsilon) m g \cos \vec{\varphi}}{\cos \vec{\varphi} \cos \vec{\alpha}+\cos \vec{\psi} \cos \bar{\beta}}, \\
\vec{f}_{2} & =\frac{(1-\varepsilon) m g \cos \vec{\psi}}{\cos \vec{\varphi} \cos \vec{\alpha}+\cos \vec{\psi} \cos \bar{\beta}}
\end{aligned}
$$


where

$$
\begin{aligned}
& \vec{\psi} \triangleq \vec{\alpha}-\bar{\theta}, \\
& \vec{\varphi} \triangleq \bar{\beta}+\bar{\theta} .
\end{aligned}
$$

Proof: Using (1), we should take

$$
\frac{\vec{f}_{1}}{\vec{f}_{2}}=\frac{\sin \bar{\beta}}{\sin \vec{\alpha}}
$$

to ensure $\ddot{x}(t)=0$. Utilizing Theorem 1 and noticing $\vec{\varphi} \triangleq \bar{\beta}+\bar{\theta}$, we get

$$
\frac{\vec{f}_{1}}{\vec{f}_{2}}=\frac{\cos (\bar{\beta}+\bar{\theta})}{\cos (\vec{\alpha}-\bar{\theta})}=\frac{\cos \vec{\varphi}}{\cos (\vec{\alpha}-\bar{\theta})}
$$

to satisfy $\ddot{y}(t)=0$ and $\ddot{\theta}(t)=0$. Putting (33) and (34) together, we should set

$$
\frac{\sin \bar{\beta}}{\sin \vec{\alpha}}=\frac{\cos \vec{\varphi}}{\cos (\vec{\alpha}-\bar{\theta})}
$$

so that the desired control parameters presented in Theorem 2 are satisfied. It is obvious that $\cos (\vec{\alpha}-\bar{\theta})$ can be rewritten as

$$
\begin{aligned}
\cos (\vec{\alpha}-\bar{\theta}) & =\cos \vec{\alpha} \cos \bar{\theta}+\sin \vec{\alpha} \sin \bar{\theta} \\
& =\sqrt{1-\sin ^{2} \vec{\alpha}} \cos \bar{\theta}+\sin \vec{\alpha} \sin \bar{\theta}
\end{aligned}
$$

where the second equality follows from noting that $\alpha(t)<\pi / 2$. Substituting (36) into (35) and rearranging, we have

$$
(\cos \vec{\varphi}-\sin \bar{\beta} \sin \bar{\theta}) \sin \vec{\alpha}=\sin \bar{\beta} \cos \bar{\theta} \sqrt{1-\sin ^{2} \vec{\alpha}}
$$

or equivalently,

$$
(\cos \vec{\varphi}-\sin \bar{\beta} \sin \bar{\theta})^{2} \sin ^{2} \vec{\alpha}=\sin ^{2} \bar{\beta} \cos ^{2} \bar{\theta}\left(1-\sin ^{2} \vec{\alpha}\right)
$$

Solving (38), we have

$$
\sin \vec{\alpha}=\frac{\sin \bar{\beta} \cos \bar{\theta}}{\sqrt{(\cos \vec{\varphi}-\sin \bar{\beta} \sin \bar{\theta})^{2}+\sin ^{2} \bar{\beta} \cos ^{2} \bar{\theta}}}
$$

which means (28) holds. Using Theorem 1 for $\alpha(t)=\vec{\alpha}$, and using (31) and (32), we obtain 
(29) and (30).

The following theorem is proposed to solve $\alpha(t), \beta(t), L_{1}(t)$ and $L_{2}(t)$.

Theorem 3: The parameters $\alpha(t), \beta(t), L_{1}(t)$ and $L_{2}(t)$ can be computed according to

$$
\begin{aligned}
\alpha(t) & =\arctan \left(\frac{L-0.5 l \cos \theta(t)-x(t)}{y(t)+0.5 l \sin \theta(t)}\right), \\
\beta(t) & =\arctan \left(\frac{x(t)-0.5 l \cos \theta(t)}{y(t)-0.5 l \sin \theta(t)}\right), \\
L_{1}(t) & =\frac{y(t)+0.5 l \sin \theta(t)}{\cos \alpha(t)} \\
L_{2}(t) & =\frac{y(t)-0.5 l \sin \theta(t)}{\cos \beta(t)} .
\end{aligned}
$$

Proof: From Fig. 1, we have

$$
\begin{aligned}
& L_{2}(t) \sin \beta(t)+l_{2}(t) \cos \theta(t)=x(t), \\
& L-L_{1}(t) \sin \alpha(t)-l_{1}(t) \cos \theta(t)=x(t), \\
& L_{2}(t) \cos \beta(t)+l_{2}(t) \sin \theta(t)=y(t), \\
& L_{1}(t) \cos \alpha(t)-l_{1}(t) \sin \theta(t)=y(t) .
\end{aligned}
$$

Using (45), (47) and (10), we derive

$$
\begin{aligned}
& L_{1}(t) \sin \alpha(t)=L-0.5 l \cos \theta(t)-x(t), \\
& L_{1}(t) \cos \alpha(t)=y(t)+0.5 l \sin \theta(t)
\end{aligned}
$$

which means that

$$
\tan \alpha(t)=\frac{L-0.5 l \cos \theta(t)-x(t)}{y(t)+0.5 l \sin \theta(t)} .
$$

Hence, equality (40) holds. Similarly, we obtain (41) by using (44), (46) and (10). Using (47) and (10), we obtain (42). From (46) and (10), it follows that equality (43) holds.

In order to obtain the desired parameters $\bar{\theta}, \bar{x}$ and $\bar{y}$ in actual operation, we propose the following Theorem to set the length $L$, and the forces $f_{1}(t)$ and $f_{2}(t)$.

Theorem 4: Under the condition that $\theta(t)=\bar{\theta}, x(t)=\bar{x}$ and $y(t)=\bar{y}$, as well as $\ddot{x}(t)=0$, $\ddot{y}(t)=0$ and $\ddot{\theta}(t)=0$, it holds that 


$$
\begin{aligned}
& \check{\beta}=\arctan \left(\frac{\bar{x}-0.5 l \cos \bar{\theta}}{\bar{y}-0.5 l \sin \bar{\theta}}\right), \\
& \check{\varphi}=\check{\beta}+\bar{\theta} \\
& \check{\alpha}=\arcsin \left(\frac{\sin \check{\beta} \cos \bar{\theta}}{\sqrt{(\cos \check{\varphi}-\sin \check{\beta} \sin \bar{\theta})^{2}+\sin ^{2} \check{\beta} \cos ^{2} \bar{\theta}}}\right), \\
& L=\bar{y} \tan \check{\alpha}+0.5 l \sin \bar{\theta} \tan \check{\alpha}+0.5 l \cos \bar{\theta}+\bar{x} \\
& \check{\psi}=\check{\alpha}-\bar{\theta}, \\
& \check{f} 1=\frac{(1-\varepsilon) m g \cos \check{\varphi}}{\cos \check{\varphi} \cos \check{\alpha}+\cos \check{\psi} \cos \check{\beta}}, \\
& \check{f}=\frac{(1-\varepsilon) m g \cos \check{\psi}}{\cos \check{\varphi} \cos \check{\alpha}+\cos \check{\psi} \cos \check{\beta}}
\end{aligned}
$$

where $\check{\beta}, \check{\varphi}, \check{\alpha}, \check{\psi}, \check{f}_{1}$ and $\check{f}_{2}$ are the values of $\beta(t), \varphi(t), \alpha(t), \psi(t), f_{1}(t)$ and $f_{2}(t)$, respectively, under the above condition.

Proof: Using (41), we have

$$
\beta(t)=\arctan \left(\frac{\bar{x}-0.5 l \cos \bar{\theta}}{\bar{y}-0.5 l \sin \bar{\theta}}\right)
$$

which means equality (49) holds. In order to ensure $\theta(t)=\bar{\theta}$ and $\beta(t)=\check{\beta}$ when $\ddot{x}(t)=0$, $\ddot{y}(t)=0$ and $\ddot{\theta}(t)=0$, we see from Theorem 2 that,

$$
\alpha(t)=\arcsin \left(\frac{\sin \check{\beta} \cos \bar{\theta}}{\sqrt{(\cos \check{\varphi}-\sin \check{\beta} \sin \bar{\theta})^{2}+\sin ^{2} \check{\beta} \cos ^{2} \bar{\theta}}}\right)
$$

with $\breve{\varphi}=\check{\beta}+\bar{\theta}$. This means equalities (50) and (51) hold. From (40), we see that

$$
L=y(t) \tan \alpha(t)+0.5 l \sin \theta(t) \tan \alpha(t)+0.5 l \cos \theta(t)+x(t) .
$$

Substituting $y(t)=\bar{y}, \alpha(t)=\check{\alpha}, \theta(t)=\bar{\theta}$ and $x(t)=\bar{x}$ into (58) to obtain (52). Using Theorem 2 for $\theta(t)=\bar{\theta}$ and $\beta(t)=\check{\beta}$, and noticing $\alpha(t)=\check{\alpha}$ in this case, we obtain (53)-(55).

Based on the above results, we design a feedback control system given as follows

$$
\dot{\chi}(t)=A \chi(t)+B(t) f(t)+d m g
$$

where the control input vector $f(t)$ including the forces $f_{1}(t)$ and $f_{2}(t)$ is designed as 


$$
f(t)=\dot{f}(t)+\check{f}
$$

with

$$
\begin{aligned}
& \dot{f}(t) \triangleq\left(\begin{array}{c}
\dot{f}_{1}(t) \\
\dot{f}_{2}(t)
\end{array}\right)=-K(t) \chi(t), \check{f} \triangleq\left(\begin{array}{c}
\check{f}_{1} \\
\check{f}_{2}
\end{array}\right), \\
& K(t)=\left(\begin{array}{llllll}
k_{11}(t) & k_{12}(t) & k_{13}(t) & k_{14}(t) & k_{15}(t) & k_{16}(t) \\
k_{21}(t) & k_{22}(t) & k_{23}(t) & k_{24}(t) & k_{25}(t) & k_{26}(t)
\end{array}\right) .
\end{aligned}
$$

Substitute (60) into (59) to get

$$
\begin{aligned}
\dot{\chi}(t) & =A \chi(t)+B(t) \dot{f}(t)+B(t) \check{f}+d m g \\
& =(A-B(t) K(t)) \chi(t)+B(t) \check{f}+d m g
\end{aligned}
$$

where the last equality uses the design $f(t)=-K(t) \chi(t)$. From (16) and (62), $A-B(t) K(t)$ can be expressed as

$$
A-B(t) K(t)=\left(h_{i j}(t)\right) \in \mathrm{R}^{6 \times 6}
$$

where

$$
\begin{aligned}
& h_{11}(t)=0, h_{12}(t)=1, h_{13}(t)=h_{14}(t)=h_{15}(t)=h_{16}(t)=0, \\
& h_{2 j}(t)=\frac{\sin \beta(t) k_{2 j}(t)-\sin \alpha(t) k_{1 j}(t)}{m}, j=1,2, \cdots, 6, \\
& h_{31}(t)=h_{32}(t)=h_{33}(t)=0, h_{34}(t)=1, h_{35}(t)=h_{36}(t)=0, \\
& h_{4 j}(t)=\frac{\cos \alpha(t) k_{1 j}(t)+\cos \beta(t) k_{2 j}(t)}{m}, \\
& h_{51}(t)=h_{52}(t)=h_{53}(t)=h_{54}(t)=h_{55}(t)=0, h_{56}(t)=1, \\
& h_{6 j}(t)=\frac{0.5 l\left(\cos \psi(t) k_{1 j}(t)-\cos \varphi(t) k_{2 j}(t)\right)}{I} .
\end{aligned}
$$

In order to control the desired parameters provided in Section II, we should design $K(t)$ such that $A-B(t) K(t)$ is stable. Hence, we hope $h_{i i}(t) \leq 0$ with $i=1,2, \cdots, 6$, and, for $i \neq j$, we hope $h_{i j}(t)=0$. From (65), we see that we can only control $\left\{h_{2 j}(t), h_{4 j}(t), h_{6 j}(t)\right\}$. As a result, we set $h_{22}(t)=\lambda_{x}(t), h_{44}(t)=\lambda_{y}(t)$ and $h_{66}(t)=\lambda_{\theta}(t)$ where $\lambda_{x}(t), \lambda_{y}(t)$ and $\lambda_{\theta}(t)$ are not positive to ensure that $A-B(t) K(t)$ are stable. Then, using (65), we have 


$$
\begin{aligned}
& \frac{\sin \beta(t) k_{22}(t)-\sin \alpha(t) k_{12}(t)}{m}=\lambda_{x}(t), \\
& \frac{\cos \alpha(t) k_{14}(t)+\cos \beta(t) k_{24}(t)}{m}=\lambda_{y}(t), \\
& \frac{0.5 l\left(\cos \psi(t) k_{16}(t)-\cos \varphi(t) k_{26}(t)\right)}{I}=\lambda_{\theta}(t) .
\end{aligned}
$$

Because the main purpose is to control $\theta(t)$, we set $h_{6 j}(t)=0$ for $j \neq 6$. Then, utilizing (65), we get

$$
\frac{0.5 l\left(\cos \psi(t) k_{1 j}(t)-\cos \varphi(t) k_{2 j}(t)\right)}{I}=0
$$

which means

$$
\cos \psi(t) k_{1 j}(t)=\cos \varphi(t) k_{2 j}(t)
$$

Solving the equation group given by (66) and (69) for $j=2$, we have

$$
\begin{aligned}
& k_{12}(t)=\frac{\cos \varphi(t) \lambda_{x}(t) m}{\sin \beta(t) \cos \psi(t)-\sin \alpha(t) \cos \varphi(t)} \\
& k_{22}(t)=\frac{\cos \psi(t) \lambda_{x}(t) m}{\sin \beta(t) \cos \psi(t)-\sin \alpha(t) \cos \varphi(t)} .
\end{aligned}
$$

Solving the equation group given by (67) and (69) for $j=4$, we have

$$
\begin{aligned}
k_{14}(t) & =\frac{\cos \varphi(t) \lambda_{y}(t) m}{\cos \alpha(t) \cos \varphi(t)+\cos \beta(t) \cos \psi(t)} \\
k_{24}(t) & =\frac{\cos \psi(t) \lambda_{y}(t) m}{\cos \alpha(t) \cos \varphi(t)+\cos \beta(t) \cos \psi(t)}
\end{aligned}
$$

In order to let $h_{4 j}(t)=0$ for $j \neq 4$, we see from (65) that we should set

$$
\cos \alpha(t) k_{1 j}(t)+\cos \beta(t) k_{2 j}(t)=0
$$

Solving the equation group given by (68) and (74) for $j=6$, we get

$$
\begin{aligned}
& k_{16}(t)=\frac{2 \cos \beta(t) \lambda_{\theta}(t) I}{l(\cos \alpha(t) \cos \varphi(t)+\cos \beta(t) \cos \psi(t))}, \\
& k_{26}(t)=-\frac{2 \cos \alpha(t) \lambda_{\theta}(t) I}{l(\cos \alpha(t) \cos \varphi(t)+\cos \beta(t) \cos \psi(t))} .
\end{aligned}
$$

Because we have obtain $k_{12}(t), k_{22}(t), k_{16}(t)$ and $k_{26}(t)$ from (70), (71), (75) and (76), respectively, in order to obtain $h_{4 j}(t)=0$ for $j \neq 4$, we can set 


$$
k_{11}(t)=k_{21}(t)=k_{13}(t)=k_{23}(t)=k_{15}(t)=k_{25}(t)=0 .
$$

Remark 3: From the above discussion presented between (59)-(77), we obtain the feedback matrix $K(t)$ where $k_{i j}(t)$ with $i=1,2$ and $j=1,2, \cdots, 6$ is given in (70)-(73) and (75)-(77). Substituting the obtained $K(t)$ into (65) and using (64), we have

$$
A-B(t) K(t)=\left(\begin{array}{cccccc}
0 & 1 & 0 & 0 & 0 & 0 \\
0 & \lambda_{x}(t) & 0 & h_{24}(t) & 0 & h_{26}(t) \\
0 & 0 & 0 & 1 & 0 & 0 \\
0 & h_{42}(t) & 0 & \lambda_{y}(t) & 0 & 0 \\
0 & 0 & 0 & 0 & 0 & 1 \\
0 & 0 & 0 & 0 & 0 & \lambda_{\theta}(t)
\end{array}\right)
$$

where

$$
\begin{aligned}
& h_{24}(t)=\frac{\sin \beta(t) \cos \psi(t)-\sin \alpha(t) \cos \varphi(t)}{\cos \alpha(t) \cos \varphi(t)+\cos \beta(t) \cos \psi(t)} \lambda_{y}(t), \\
& h_{26}(t)=-\frac{2 I(\sin \alpha(t) \cos \beta(t)+\sin \beta(t) \cos \alpha(t))}{l m(\cos \psi(t) \cos \beta(t)+\cos \alpha(t) \cos \varphi(t))} \lambda_{\theta}(t), \\
& h_{42}(t)=\frac{\cos \alpha(t) \cos \varphi(t)+\cos \beta(t) \cos \psi(t)}{\sin \beta(t) \cos \psi(t)-\sin \alpha(t) \cos \varphi(t)} \lambda_{x}(t) .
\end{aligned}
$$

Remark 4: Using (78) and the Laplace expansion, we have

$$
\begin{aligned}
\left|\lambda(t) I_{6}-(A-B(t) K(t))\right| & \\
= & \lambda(t)^{3}\left(\lambda(t)-\lambda_{\theta}(t)\right)\left(\left(\lambda(t)-\lambda_{x}(t)\right)\left(\lambda(t)-\lambda_{y}(t)\right)\right. \\
& \left.\quad-h_{24}(t) h_{42}(t)\right) \\
= & \lambda(t)^{3}\left(\lambda(t)-\lambda_{\theta}(t)\right)\left(\lambda(t)^{2}-\left(\lambda_{x}(t)+\lambda_{y}(t)\right) \lambda(t)\right. \\
& \left.+\lambda_{x}(t) \lambda_{y}(t)-h_{24}(t) h_{42}(t)\right) .
\end{aligned}
$$

From (79) and (81), it follows that

$$
h_{24}(t) h_{42}(t)=\lambda_{x}(t) \lambda_{y}(t) .
$$

Substitute (83) into (82) to get 


$$
\begin{aligned}
\left|\lambda(t) I_{6}-(A-B(t) K(t))\right| & =\lambda(t)^{3}\left(\lambda(t)-\lambda_{\theta}(t)\right)\left(\lambda(t)^{2}-\left(\lambda_{x}(t)+\lambda_{y}(t)\right) \lambda(t)\right) \\
& =\lambda(t)^{4}\left(\lambda(t)-\lambda_{\theta}(t)\right)\left(\lambda(t)-\left(\lambda_{x}(t)+\lambda_{y}(t)\right)\right) .
\end{aligned}
$$

From (84), we see that the eigenvalues of the matrix $A-B(t) K(t)$ are less than or equal to zero if $\lambda_{\theta}(t)$ and $\lambda_{x}(t)+\lambda_{y}(t)$ are less than or equal to zero. Hence, we can set the values of $\lambda_{\theta}(t)$ and $\lambda_{x}(t)+\lambda_{y}(t)$ to ensure that $A-B(t) K(t)$ is stable.

We now begin to design $\lambda_{\theta}(t), \lambda_{x}(t)$ and $\lambda_{y}(t)$. From (63) and (78)-(81), we see that

$$
\dot{\chi}(t)=A \chi(t)+B(t) \check{f}+d m g
$$

if $\lambda_{\theta}(t)=\lambda_{x}(t)=\lambda_{y}(t)=0$. Then, we can use $\check{f}$ to obtain the desired values presented in Theorem 4 if we have $\theta(t)=\bar{\theta}, x(t)=\bar{x}, y(t)=\bar{y}$ and $\dot{v}_{\theta}=\dot{v}_{x}=\dot{v}_{y}=0$ when $\lambda_{\theta}(t)=\lambda_{x}(t)=\lambda_{y}(t)=0$. Hence, we design $\lambda_{\theta}(t), \lambda_{x}(t)$ and $\lambda_{y}(t)$ using the following strategy

$$
\begin{aligned}
& \lambda_{\theta}(t)=-k_{\theta}(t)|\theta(t)-\bar{\theta}|-k_{\theta}^{\triangleleft}(t)\left|\dot{v}_{\theta}(t)\right|, \\
& \lambda_{x}(t)=-k_{x}(t)|x(t)-\bar{x}|-k_{x}^{\triangleleft}(t)\left|\dot{v}_{x}(t)\right|, \\
& \lambda_{y}(t)=-k_{y}(t)|y(t)-\bar{y}|-k_{y}^{\triangleleft}(t)\left|\dot{v}_{y}(t)\right|
\end{aligned}
$$

where $k_{\theta}(t) \geq 0, k_{\theta}^{\triangleleft}(t) \geq 0, k_{x}(t) \geq 0, k_{x}^{\triangleleft}(t) \geq 0, k_{y}(t) \geq 0$ and $k_{y}^{\triangleleft}(t) \geq 0$.

Remark 5: From (86)-(88), we see that the strategy for designing $\lambda_{\theta}(t), \lambda_{x}(t)$ and $\lambda_{y}(t)$ can guarantee that $\lambda_{\theta}(t), \lambda_{x}(t)$ and $\lambda_{y}(t)$ are all less than or equal to zero. Then, we see from Remark 4 that $A-B(t) K(t)$ is stable. Also, we have $\theta(t)=\bar{\theta}, x(t)=\bar{x}, y(t)=\bar{y}$ and $\dot{v}_{\theta}=\dot{v}_{x}=\dot{v}_{y}=0$ when $\lambda_{\theta}(t)=\lambda_{x}(t)=\lambda_{y}(t)=0$. When the feedback control system is in such case, using Theorem 4 , we have $\beta(t)=\check{\beta}$ and $\alpha(t)=\check{\alpha}$. Substituting $\theta(t)=\bar{\theta}, \beta(t)=\check{\beta}$ and $\alpha(t)=\check{\alpha}$ into $B(t)$ defined in (16), as well as using (6), (8), (50) and (53), we have $B(t)=\check{B}$ where

$$
\check{B}=\left(\begin{array}{cc}
0 & 0 \\
\sin \check{\alpha} / m & -\sin \check{\beta} / m \\
0 & 0 \\
-\cos \check{\alpha} / m & -\cos \check{\beta} / m \\
0 & 0 \\
-0.5 l \cos \check{\psi} / I & 0.5 l \cos \check{\varphi} / I
\end{array}\right) .
$$

Then, we see from Theorem 4 that the forces $\check{f}_{1}$ and $\check{f}_{2}$ can ensure the desired values presented 
in Theorem 4.

Based on the above discussion, we propose the following nonlinear state feedback control algorithm so that the requirements given in Section II are satisfied.

\section{Nonlinear State Feedback Control Algorithm}

Initialization: Given $\bar{\theta}, \bar{x}, \bar{y}$ and $\varepsilon$ with $0 \leq \varepsilon \leq 1$ where they can obtain from the user's clinical and physical circumstances.

Step 1: Compute $L, \check{f}_{1}$ and $\check{f}_{2}$ according to (49)-(55).

Step 2: Compute $\alpha(t), \beta(t), \varphi(t)$ and $\psi(t)$ utilizing (40),(41), (6) and (8) in sequence.

Step 3: Compute $\lambda_{\theta}(t), \lambda_{x}(t)$ and $\lambda_{y}(t)$ via (86), (87) and (88), respectively, where $k_{\theta}(t) \geq 0$, $k_{\theta}^{\triangleleft}(t) \geq 0, k_{x}(t) \geq 0, k_{x}^{\triangleleft}(t) \geq 0, k_{y}(t) \geq 0$ and $k_{y}^{\triangleleft}(t) \geq 0$, which are selected according to the user's clinical and physical circumstances.

Step 4: Compute $k_{12}(t), k_{22}(t), k_{14}(t), k_{24}(t), k_{16}(t)$ and $k_{26}(t)$ in terms of (70), (71), (72), (73), (75) and (76), respectively.

Step 5: Compute $\check{f}_{1}(t)$ and $\check{f}_{2}(t)$ according to

$$
\begin{aligned}
& f_{1}(t)=-k_{12}(t) v_{x}(t)-k_{14}(t) v_{y}(t)-k_{16}(t) v_{\theta}(t) \\
& f_{2}(t)=-k_{22}(t) v_{x}(t)-k_{24}(t) v_{y}(t)-k_{26}(t) v_{\theta}(t)
\end{aligned}
$$

Step 6: Compute $f_{1}(t)$ and $f_{2}(t)$ according to

$$
\begin{aligned}
& f_{1}(t)= \begin{cases}f_{1}^{\nabla}, & \hat{f}_{1}(t)+\check{f}_{1} \geq f_{1}^{\nabla} ; \\
f_{1}(t)+\check{f}_{1}, & 0<\tilde{f}_{1}(t)+\check{f}_{1}<f_{1}^{\nabla} ; \\
0, & \tilde{f}_{1}(t)+\check{f}_{1} \leq 0,\end{cases} \\
& f_{2}(t)= \begin{cases}f_{2}^{\nabla}, & f_{2}(t)+\check{f}_{2} \geq f_{2}^{\nabla} ; \\
f_{2}(t)+\check{f}_{2}, & 0<\tilde{f}_{2}(t)+\check{f}_{2}<f_{2}^{\nabla} ; \\
0, & f_{2}(t)+\check{f}_{2} \leq 0 .\end{cases}
\end{aligned}
$$

Proof: It is obvious that we need to prove Step 5 and Step 6. Substituting (62) and the 
definition of $\chi(t)$ given in (14) into $\left(\begin{array}{l}f_{1}(t) \\ f_{2}(t)\end{array}\right)=-K(t) \chi(t)$, as well as using (77), we prove Step 5. Using the definition of $f(t)$ given in (14), the definition of $f(t)$ and $\check{f}$ given in (61), and using (60), we have

$$
f_{1}(t)=\check{f}_{1}(t)+\check{f}_{1}, f_{2}(t)=\check{f}_{2}(t)+\check{f}_{2}
$$

Then, using (94), as well as considering the requirement that $0 \leq f_{1}(t) \leq f_{1}^{\nabla}$ and $0 \leq f_{2}(t) \leq f_{2}^{\nabla}$ presented in Section II, we prove Step 6.

Remark 6: In this paper, the pulling force $f_{i}(t)$ with $i=1,2$ is designed as $f_{i}(t)=\tilde{f}_{i}(t)+\check{f}_{i}$, that is, $f_{i}(t)$ includes two parts $f_{i}(t)$ and $\check{f}_{i}$, where $f_{i}(t)$ can guarantee the stability of the feedback control system, and control the parameters to be close to the desired values. When the desired parameters are reached, $f_{i}(t)$ no longer has any effect on the system, namely, $f_{i}(t)=0$, and the force $\breve{f}_{i}$ can guarantee that the system keeps the desired parameters.

Remark 7: From (86), we find that the values $k_{\theta}(t)$ and $k_{\theta}^{\triangleleft}(t)$ have influences on $\lambda_{\theta}(t)$. Especially, $k_{\theta}(t)$ or $k_{\theta}^{\triangleleft}(t)$ largely affect $\lambda_{\theta}(t)$ when $|\theta(t)-\bar{\theta}|$ or $\left|\dot{v}_{\theta}(t)\right|$ has a large value. Hence, in order to restrict the value $\lambda_{\theta}(t)$ so that $\left|\lambda_{\theta}(t)\right|$ is not too large, we need to set some limits for the values of $k_{\theta}(t)$ and $k_{\theta}^{\triangleleft}(t)$. A strategy for selecting the values of $k_{\theta}(t)$ and $k_{\theta}^{\triangleleft}(t)$ is that we take the value of $k_{\theta}(t)$ according to the value of $|\theta(t)-\bar{\theta}|$, and take the value of $k_{\theta}^{\triangleleft}(t)$ according to the value of $\left|\dot{v}_{\theta}(t)\right|$. We see from (87) and (88) that we can select the values of $k_{x}(t)$ and $k_{x}^{\triangleleft}(t)$, as well as $k_{y}(t)$ and $k_{y}^{\triangleleft}(t)$ in the same way.

\section{Simulation EXAmple}

In this section, a simulation example is given to demonstrate the performance of the proposed nonlinear state feedback control algorithm.

Consider a user with the mass $m=75 \mathrm{~kg}$, the gravitational acceleration $g=9.81 \mathrm{~m} / \mathrm{s}^{2}$, the shoulder width $l=0.5 \mathrm{~m}$ and the moment of inertia $I=20.3 \mathrm{~kg} \cdot \mathrm{m}^{2}$. The maximum control input is $(450 \mathrm{~N}, 450 \mathrm{~N})^{\mathrm{T}}$, that is, $f_{1}^{\nabla}=450 \mathrm{~N}$ and $f_{2}^{\nabla}=450 \mathrm{~N}$. Considering that $y(t)$ does not have large change, it is reasonable to assume that $y(t)$ is constant and $v_{y}(t)=0$. Then, the aim becomes to control $\theta(t)$ and $x(t)$ with constant $y(t)$ and $v_{y}(t)=0$. To test the performance of the proposed control algorithm, we take three cases for different combinations of the parameter values, which is given as follows:

Case 1: $\bar{\theta}=\frac{\pi}{9} \mathrm{rad}, \bar{x}=1.3 \mathrm{~m}$ and $\varepsilon=0.8 ;$ Case 2: $\bar{\theta}=\frac{\pi}{8} \mathrm{rad}, \bar{x}=1.4 \mathrm{~m}$ and $\varepsilon=0.7$; 
Case 3: $\bar{\theta}=\frac{\pi}{10} \mathrm{rad}, \bar{x}=0.9 \mathrm{~m}$ and $\varepsilon=0.9$.

In this example, because we only need to control $\theta(t)$ and $x(t)$, we set $k_{y}(t)=0$ and $k_{y}^{\triangleleft}(t)=0$, and we use the following strategy for choosing $k_{\theta}(t), k_{\theta}^{\triangleleft}(t), k_{x}(t), k_{x}^{\triangleleft}(t)$ :

$$
\begin{aligned}
& k_{\theta}(t)= \begin{cases}8000, & |\theta(t)-\bar{\theta}|<\frac{\pi}{14400} \mathrm{rad} ; \\
1600, & \frac{\pi}{14400} \leq|\theta(t)-\bar{\theta}|<\frac{\pi}{1440} \mathrm{rad} ; \\
\frac{4}{|\theta(t)-\bar{\theta}|}, & \frac{\pi}{144} \leq|\theta(t)-\bar{\theta}|<\frac{\pi}{72} \mathrm{rad} ; \\
0.2, & \frac{\pi}{72} \leq|\theta(t)-\bar{\theta}|<\frac{\pi}{18} \mathrm{rad} ; \\
\frac{0.2}{|\theta(t)-\bar{\theta}|}, & |\theta(t)-\bar{\theta}| \geq \frac{\pi}{18} \mathrm{rad},\end{cases} \\
& k_{\theta}^{\triangleleft}(t)= \begin{cases}40, & \left|\dot{v}_{\theta}(t)\right|<\frac{\pi}{1440} \mathrm{rad} / \mathrm{s} ; \\
\frac{10,}{40,} & \frac{\pi}{1440} \leq\left|\dot{v}_{\theta}(t)\right|<\frac{\pi}{144} \mathrm{rad} / \mathrm{s} ; \\
3, & \frac{\pi}{144} \leq\left|\dot{v}_{\theta}(t)\right|<\frac{\pi}{72} \mathrm{rad} / \mathrm{s} ; \\
0.2, & \frac{\pi}{72} \leq\left|\dot{v}_{\theta}(t)\right|<\frac{\pi}{18} \mathrm{rad} / \mathrm{s} ; \\
\frac{0.1}{\left|\dot{v}_{\theta}(t)\right|}, & \left|\dot{v}_{\theta}(t)\right| \geq \frac{\pi}{18} \mathrm{rad} / \mathrm{s}, \\
0.01, & |x(t)-\bar{x}|<0.0001 \mathrm{~m} ; \\
\frac{0.002}{|x(t)-\bar{x}|}, & 0.0001 \leq|x(t)-\bar{x}|<0.001 \mathrm{~m} ; \\
0.002, & 0.001 \leq|x(t)-\bar{x}|<0.01 \mathrm{~m} ; \\
\frac{0.001}{|x(t)-\bar{x}|}, & 0.01 \leq|x(t)-\bar{x}|<0.05 \mathrm{~m} ; \\
0.001, & 0.05 \leq|x(t)-\bar{x}|<0.2 \mathrm{~m} ; \\
\frac{0.001}{|x(t)-\bar{x}|}, & |x(t)-\bar{x}| \geq 0.2 \mathrm{~m}, \\
0.002, & 0.005 \leq\left|\dot{v}_{\theta}(t)\right|<0.05 \mathrm{~m} / \mathrm{s} ; \\
0.0005, & 0.05 \leq\left|\dot{v}_{\theta}(t)\right|<0.2 \mathrm{~m} / \mathrm{s} ; \\
\frac{0.0005}{\left|\dot{v}_{x}(t)\right|}, & \left|\dot{v}_{x}(t)\right| \geq 0.2 \mathrm{~m} / \mathrm{s} .\end{cases} \\
& \begin{array}{ll}
0.005, & \left|\dot{v}_{\theta}(t)\right|<0.0005 \mathrm{~m} / \mathrm{s} ;
\end{array}
\end{aligned}
$$




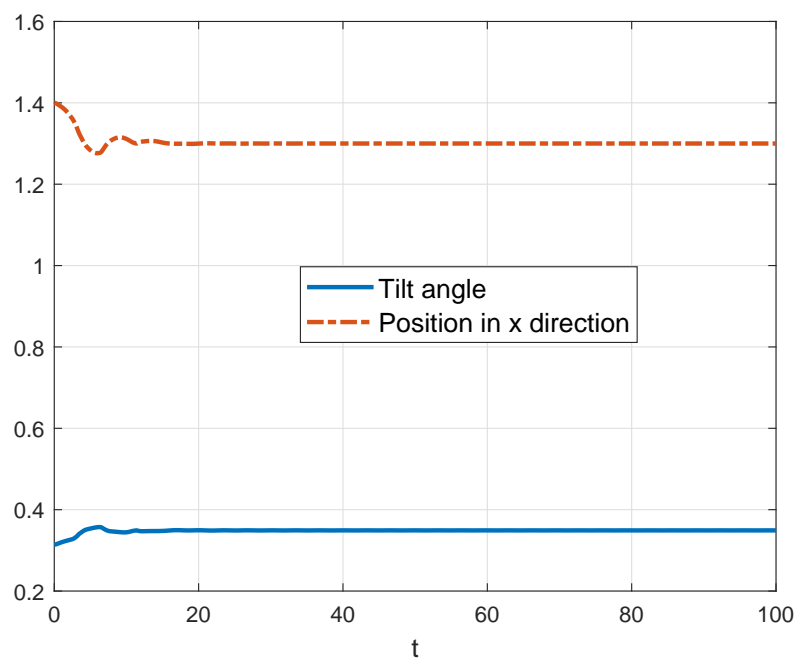

Fig. 2. Tilt angle $\theta(t)$ and position $x(t)$ for Case 1 .

The above strategy for choosing $k_{\theta}(t), k_{\theta}^{\triangleleft}(t), k_{x}(t)$ and $k_{x}^{\triangleleft}(t)$ is based on Remark 7, (86) and (87). For each case, we select different initial values $\theta(0), v_{\theta}(0)=0, x(0)$ and $v_{x}(0)$, as well as $y(t)$ and $v_{y}(t)$, which is provided as follows:

Case 1: $\theta(0)=\frac{\pi}{10} \mathrm{rad}, v_{\theta}(0)=0 \mathrm{rad} / \mathrm{s}, x(0)=1.4 \mathrm{~m}$,

$$
v_{x}(0)=0 \mathrm{~m} / \mathrm{s}, y(t)=1.1 \mathrm{~m}, v_{y}(t)=0 \mathrm{~m} / \mathrm{s} ;
$$

Case 2: $\theta(0)=\frac{\pi}{9} \mathrm{rad}, v_{\theta}(0)=0 \mathrm{rad} / \mathrm{s}, x(0)=1.5 \mathrm{~m}$,

$$
v_{x}(0)=0 \mathrm{~m} / \mathrm{s}, y(t)=1.1 \mathrm{~m}, v_{y}(t)=0 \mathrm{~m} / \mathrm{s}
$$

Case 3: $\theta(0)=\frac{\pi}{9} \mathrm{rad}, v_{\theta}(0)=0 \mathrm{rad} / \mathrm{s}, x(0)=1 \mathrm{~m}$,

$$
v_{x}(0)=0 \mathrm{~m} / \mathrm{s}, y(t)=1.2 \mathrm{~m}, v_{y}(t)=0 \mathrm{~m} / \mathrm{s} .
$$

The tilt angle $\theta(t)$ and the position $x(t)$ for Case 1, Case 2 and Case 3 are given in Fig. 2, Fig. 3 and Fig. 4, respectively. From observing Figs. 2-4, we find that, for the three cases of different parameter values, the tilt angle $\theta(t)$ approaches $\bar{\theta}$ and the position $x(t)$ approaches $\bar{x}$, which indicates the effectiveness of the proposed nonlinear state feedback control algorithm. Figs. 2-4 also shows that, each case takes a different amount of time to reach the desired parameter values $\bar{\theta}$ and $\bar{x}$ where Case 1 needs the shortest amount of time to reach the desired parameter values, and Case 3 needs the longest amount of time to reach the desired parameter values. 


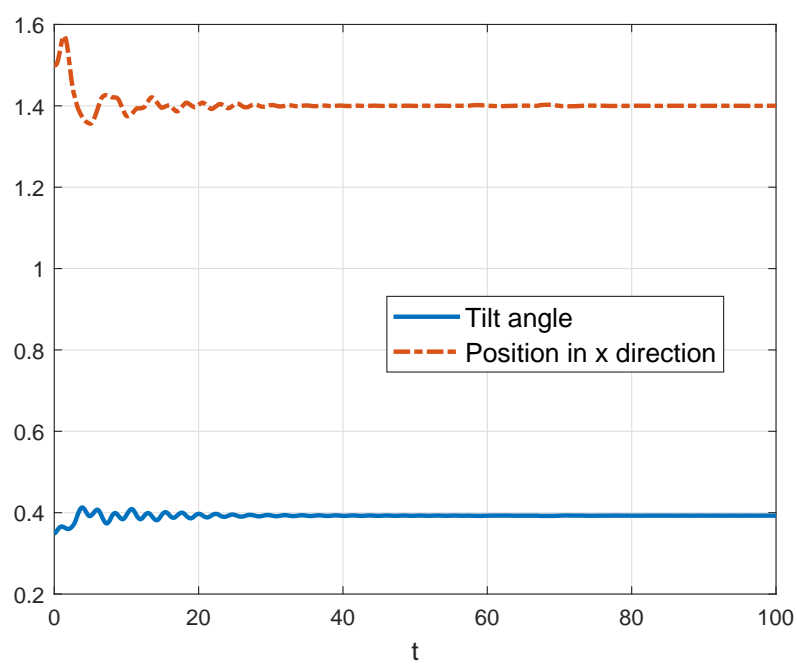

Fig. 3. Tilt angle $\theta(t)$ and position $x(t)$ for Case 2 .

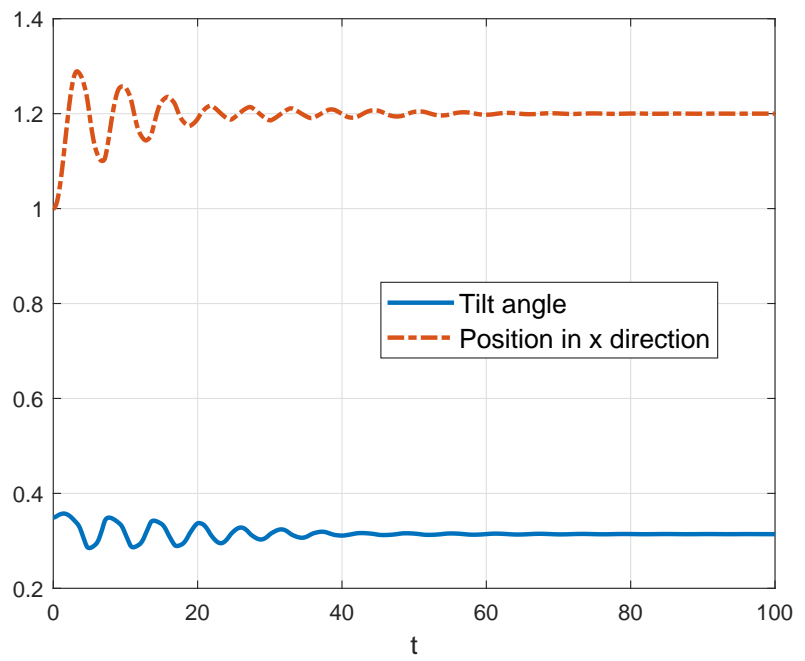

Fig. 4. Tilt angle $\theta(t)$ and position $x(t)$ for Case 3 .

\section{Conclusion}

A nonlinear dynamical system in state space form with control input has been established to model a novel WATR. Based on the nonlinear dynamical system, a feedback control system has been developed, and a nonlinear state feedback control algorithm has been designed. The control input consists of two parts, that is, a dynamical part and a constant part. The dynamical part in the control input guarantees the stability of the feedback control system, and drives the user's tilt angle and position to approach the desired values. The constant part ensures that the desired values are kept when the user's tilt angle and position reach the desired values. A simulation 
example was provided, and the simulation results have shown that, for different initial values, the proposed state feedback control algorithm can guarantee the user's tilt angle and position to approach the desired values under some limits for the control input.

\section{REFERENCES}

[1] L. L. Haubert, D. D. Gutierrez, C. J. Newsam, J. K. Gronley, S. J. Mulroy, and J. Perry, "A comparison of shoulder joint forces during ambulation with crutches versus a walker in persons with incomplete spinal cord injury", Arch. Phys. Med. Rehabil., vol. 87, no. 1, pp. 63-70, Jan. 2006.

[2] P. R. Culmer, P. C. Brooks, D. N. Strauss, D. H. Ross, M. C. Levesley, R. J. O’Connor, and B. B. Bhakta, "An instrumented walking aid to assess and retrain gait", IEEE/ASME Trans. Mechatronics, vol. 19, no. 1, pp. 141-148, Feb. 2014.

[3] B. N. Fournier, E. D. Lemaire, A. J. J. Smith, and M. Doumit, "Modeling and simulation of a lower extremity powered exoskeleton”, IEEE Trans. Neural Syst. Rehabil. Eng., vol. 26, no. 8, pp. 1596-1603, Aug. 2018.

[4] D. C. Dang and Y. S. Suh, "Walking distance estimation using walking canes with inertial sensors", Sensors, vol. 18, no. 1, p. 230, Jan. 2018.

[5] E. Dorschky, M. Nitschke, A. K. Seifer, A. J. Bogert, and B. M. Eskofier, "Estimation of gait kinematics and kinetics from inertial sensor data using optimal control of musculoskeletal models", J. Biomech., vol. 95, no. 11, 109278, Oct. 2019.

[6] D. Pinto-Fernandez, D. Torricelli, M. C. Sanchez-Villamanan, F. Aller, K. Mombaur, R. Conti, N. Vitiello, J. C. Moreno, and J. L. Pons, "Performance evaluation of lower limb exoskeletons: a systematic review", IEEE Trans. Neural Syst. Rehabil. Eng., vol. 28, no. 7, pp. 1573-1583, Jul. 2020.

[7] H. Yu, M. Spenko, and S. Dubowsky, "An adaptive shared control system for an intelligent mobility aid for the elderly", Auton. Robot., vol. 15, no. 1, pp. 53-66, Jul. 2003.

[8] Y. Hirata, A. Hara, and K. Kosuge, "Motion control of passive intelligent walker using servo brakes", IEEE Trans. Robot., vol. 23, no. 5, pp. 981-990, Oct. 2007.

[9] G. Lee, T. Ohnuma, N. Y. Chong, and S. G. Lee, "Walking intent-based movement control for JAIST active robotic walker", IEEE Trans. Syst. Man Cybern., Syst., vol. 44, no. 5, pp. 665-672, May 2014.

[10] H. M. Gross, A. Scheidig, K. Debes, E. Einhorn, M. Eisenbach, S. Mueller, T. Schmiedel, T. Q. Trinh, C. Weinrich, T. Wengefeld, A. Bley, and C. Martin, "ROREAS: robot coach for walking and orientation training in clinical post-stroke rehabilitation-prototype implementation and evaluation in field trials”, Auton. Robot., vol. 41, no. 3, pp. 679-698, Mar. 2017.

[11] M. Andreetto, S. Divan, F. Ferrari, D. Fontanelli, L. Palopoli, and D. Prattichizzo, "Combining haptic and bang-bang braking actions for passive robotic walker path following”, IEEE Trans. Haptics, vol. 12, no. 4, pp. 542-553, Oct.-Dec. 2019.

[12] B. A. Slavens, N. Bhagchandani, M. Wang, P. A. Smith, and G. F. Harris, "An upper extremity inverse dynamics model for pediatric Lofstrand crutch-assisted gait”, J. Biomech., vol. 44, no. 11, pp. 2162-2167, Jul. 2011.

[13] E. Sardini, M. Serpelloni, and M. Lancini, "Wireless instrumented crutches for force and movement measurements for gait monitoring”, IEEE Trans. Instrum. Meas., vol. 64, no. 12, pp. 3369-3379, Dec. 2015.

[14] F. L. Haufe, R. H. Hassani, R. Riener, and P. Wolf, "The insight crutches: analyzing the role of arm support during robot-assisted leg movements", IEEE Robot. Autom. Mag., vol. 27, no. 1, pp. 103-113, Mar. 2020.

[15] K. Wakita, J. Huang, P. Di, K. Sekiyama, and T. Fukuda, "Human-walking-intention-based motion control of an omnidirectional-type cane robot”, IEEE/ASME Trans. Mechatronics, vol. 18, no. 1, pp. 285-296, Feb. 2013.

[16] P. V. Lam and Y. Fujimoto, "A robotic cane for balance maintenance assistance", IEEE Trans. Ind. Informat., vol. 15, no. 7, pp. 3998-4009, Jul. 2019. 
[17] K. Kong and D. Jeon, "Design and control of an exoskeleton for the elderly and patients", IEEE/ASME Trans. Mechatronics, vol. 11, no. 4, pp. 428-432, Aug. 2006.

[18] T. Kagawa, H. Ishikawa, T. Kato, C. Sung, and Y. Uno, "Optimization-based motion planning in joint space for walking assistance with wearable robot”, IEEE Trans. Robot., vol. 31, no. 2, pp. 415-424, Apr. 2015.

[19] K. H. Ha, S. A. Murray, and M. Goldfarb, "An approach for the cooperative control of FES with a powered exoskeleton during level walking for persons with paraplegia”, IEEE Trans. Neural Syst. Rehabil. Eng., vol. 24, no. 4, pp. 455-466, Apr. 2016.

[20] M. Hamaya, T. Matsubara, T. Noda, T. Teramae, and J. Morimoto, "Learning assistive strategies for exoskeleton robots from user-robot physical interaction”, Pattern Recognit. Lett., vol. 99, no. 1, pp. 67-76, Nov. 2017.

[21] X. Wu, D. Liu, M. Liu, C. Chen, and H. Guo, "Individualized gait pattern generation for sharing lower limb exoskeleton robot", IEEE Trans. Autom. Sci. Eng., vol. 15, no. 4, pp. 1459-1470, Oct. 2018.

[22] Y. Hirata, T. Baba, and K. Kosuge, "Motion control of omni-directional type walking support system "walking helper"”, in Proc. IEEE Workshop Robot Human Interactive Commun., Millbrae, USA, Oct. 2003, pp. 85-90.

[23] M. M. Martins, C. P. Santos, A. Frizera-Neto, and R. Ceres, "Assistive mobility devices focusing on smart walkers: Classification and reviews", Robot. Autonom. Syst., vol. 60, no. 4, pp. 548-562, Apr. 2012.

[24] S. Y. Kim, L. Yang, I. J. Park, E. J. Kim, M. S. J. Park, S. H. You, Y. H. Kim, H. Y. Ko, and Y. I. Shin, "Effects of innovative WALKBOT robotic-assisted locomotor training on balance and gait recovery in hemiparetic stroke: a prospective, randomized, experimenter blinded case control study with a four-week follow-up”, IEEE Trans. Neural Syst. Rehabil. Eng., vol. 23, no. 4, pp. 636-642, Jul. 2015.

[25] C. D. Lim, C. M. Wang, C. Y. Cheng, Y. Chao, S. H. Tseng, and L. C. Fu, "Sensory cues guided rehabilitation robotic walker realized by depth image-based gait analysis", IEEE Trans. Autom. Sci. Eng., vol. 13, no. 1, pp. 171-180, Jan. 2016.

[26] J. Li, B. Shen, C. M. Chew, C. L. Teo, and A. N. Poo, "Novel functional task-based gait assistance control of lower extremity assistive device for level walking”, IEEE Trans. Ind. Electron., vol. 63, no. 2, pp. 1096-1106, Feb. 2016.

[27] H. Chang, P. Sun, and S. Wang, "A robust adaptive tracking control method for a rehabilitative walker using random parameters", Int. J. Control, vol. 90, no. 7, pp. 1446-1456, Jul. 2017.

[28] D. H. Lee, S. S. Lee, C. K. Ahn, P. Shi, and C. C. Lim, "Finite distribution estimation-based dynamic window approach to reliable obstacle avoidance of mobile robot”, IEEE Trans. Ind. Electron., vol. 68, no. 10, pp. 9998-10006, Oct. 2021.

[29] P. Shi, M. Liu, and L. Zhang, "Fault-tolerant sliding-mode-observer synthesis of Markovian jump systems using quantized measurements", IEEE Trans. Ind. Electron., vol. 62, no. 9, pp. 5910-5918, Sep. 2015.

\section{STATEMENTS \& DECLARATIONS}

\section{Funding}

This work was partially supported by the National Nature Science Foundation of China (62073125, 61773131), the DongGuan Innovative Research Team Program (2020607202006), and the Australian Research Council (DP170102644).

\section{Competing Interests}

The authors have no relevant financial or non-financial interests to disclose. 


\section{Author Contributions}

All authors read and approved the final manuscript. The concrete contributions of each author are given as follows:

Wei Liu: Formal analysis, Methodology, Software, Writing-original draft, Writing-review \& editing

Shuoyu Wang: System modeling, Supervision, Review

Peng Shi: Supervision, Review

\section{Data Availability}

The manuscript has no associated data. 